\title{
Competing Risk Nomograms Predicting Cause-specific Mortality and Competing Mortality in Patients with Lung Adenosquamous Carcinoma
}

\section{Xiao Wu}

Zhejiang University School of Medicine First Affiliated Hospital

Wenfeng Yu

Zhejiang University School of Medicine First Affiliated Hospital

\section{RH Petersen}

Copenhagen university hospital

\section{Hongxu Sheng}

Zhejiang University School of Medicine First Affiliated Hospital

\section{Yiqing Wang}

Zhejiang University School of Medicine First Affiliated Hospital

Wang Lv

Zhejiang University School of Medicine First Affiliated Hospital

Jian Hu ( $\nabla$ dr_hujian@zju.edu.cn )

Zhejiang University School of Medicine First Affiliated Hospital https://orcid.org/0000-0002-9494-9828

\section{Research article}

Keywords: Adenosquamous carcinoma, Lung cancer, competing-risk analysis, Cumulative incidence, Nomogram.

Posted Date: March 4th, 2020

DOI: https://doi.org/10.21203/rs.3.rs-15998/v1

License: (c) (i) This work is licensed under a Creative Commons Attribution 4.0 International License. Read Full License

Version of Record: A version of this preprint was published at BMC Cancer on May 16th, 2020. See the published version at https://doi.org/10.1186/s12885-020-06927-w. 


\section{Abstract}

Background Adenosquamous carcinoma (ASC) is an uncommon histological subtype of lung cancer. The purpose of this study was to assess the cumulative incidence of lung cancer-specific mortality (LC-SM) and other cause-specific mortality (OCSM) for lung ASC patients, and construct the corresponding competing risk nomograms. Methods Data on 2,705 patients with first primary lung ASC histologically diagnosed during 2004 and 2015 were extracted from the Surveillance, Epidemiology, and End Results (SEER) database. Cumulative incidence function (CIF) was utilized to calculate the 3-year and 5-year probabilities of LC-SM and OCSM, and a competing risk model was built. Based on the model, we developed the competing risk nomograms, and the corresponding concordance indexes ( $\mathrm{C}$-index) and calibration curves were derived to assess the model performance. Results The 3-year and 5-year cumulative mortalities were $49.6 \%$ and $55.8 \%$ for LC-SM, and $8.2 \%$ and $11.8 \%$ for OCSM, respectively. In multivariate analysis, increasing age, male sex, no surgery, and advanced T, N and $\mathrm{M}$ stage were related to a significantly higher likelihood of LC-SM, in contrast with increasing age and surgery served as predictors for an elevated risk of OCSM. The nomogram showed a good calibration, and the 5-year C-indexes for predicting probabilities of LC-SM and OCSM in the validation cohort were 0.79 and 0.63 , respectively. Conclusions The competing risk nomogram displayed an excellent discrimination and calibration, especially for predicting LC-SM. With the aid of this individualized predictive tool, it is more expedient for clinicians to devise appropriate treatment protocols and follow-up schedules.

\section{Background}

Adenosquamous carcinoma (ASC), a rare histological subtype of lung cancer, occupies less than $3 \%$ of all lung cancer cases [1-4]. In general, ASC is defined as a carcinoma with each adenocarcinoma (ADC) components and squamous cell carcinoma (SCC) components exceeding $10 \%$ of the entire tumor [5]. There is a substantial difference between ASC and other histological subtypes of lung cancer in the clinicopathological characteristics. ASC patients are more likely to present with a larger tumor size, a higher frequency of lymphatic/ pleural invasion, and a worse histological grade than ADC and SCC patients $[3,6]$. In terms of survival, ASC patients share an unfavorable prognosis compared to ADC and SCC patients $[3,6,7]$.

A majority of ASC patients are diagnosed at an age over 60 years [3, 6, 7], and elderly patients tend to have a higher prevalence of comorbidity than younger patients [8]. In addition, comorbidity has been proved to be a strong predictor of other cause-specific mortality (OCSM) in various cancers [9-11]. In light of the high risk of OCSM, it is essential to take OCSM into account when performing survival analysis for lung ASC. Meanwhile, in the presence of competing events (such as OCSM), a traditional Cox proportional hazard model is no longer suitable, as it ignores the existence of competing risks, which may inevitably overestimate the incidence of cancer-specific mortality [12]. In this context, competing risk model is superior to the conventional Cox model, because it takes into consideration the competing events and can differentiate between the effects of therapy and risk factors on specific events [12, 13]. However, to date, there are no studies adopting a competing risk model to examine the influencing factors for the prognosis of patients with lung ASC.

In addition, nomogram, a visual display of the linear prediction model, is capable of calculating the individual risk probabilities of a clinical event based on the predictive variables on the graph [14]. Due to the usefulness of a nomogram, it has been extensively applied to various cancers, such as non-small-cell Lung Cancer (NSCLC) [15], hepatocellular carcinoma [16], and breast cancer [17]. However, to our knowledge, there are no studies aiming to develop a nomogram to predict the survival for lung ASC patients, using a competing risk regression model.

Therefore, a competing risk analysis was performed to determine the predictive factors of the lung cancer-specific mortality (LC-SM) and OCSM for patients with lung ASC. We developed nomograms to offer clinicians a quantitative 
means to assess the individual cumulative incidences of LC-SM and OCSM to improve clinical decision making.

\section{Methods}

\section{Data sources}

Data on patients with first primary lung ASC histologically diagnosed between 2004 and 2015 were extracted from the Surveillance, Epidemiology, and End Results (SEER) registries (1975-2016 dataset). The study population comprised patients with the following International Classification of Diseases for Oncology, Third Edition (ICD-0-3): site code (C340-C349) and histological code (8560/3). The study time span was set from 2004 to 2015 on the basis of the first year of the American Joint Committee on Cancer (AJCC) 6th edition (2004+) and a minimum of one-year follow-up.

Related demographic and clinicopathological variables were collected, including age, gender, race, marital status, tumor laterality, tumor site, histologic grade, TNM stage, surgical treatment, survival time, and causes of death. We excluded the patients with any unknown variable values mentioned above. Age was categorized as $<65$ years and $\geq 65$ years; marital status was divided into unmarried and married; histological grade was classified into grade I (well differentiated), grade II (moderately differentiated), grade III (poorly differentiated), and grade IV (undifferentiated); causes of death were categorized as alive, LC-SM and OCSM. As no radiation and unknown radiation have been merged into "None/Unknown" since 2016, and there was a substantial heterogeneity in chemotherapy, we did not include radiation and chemotherapy into the present study. The detailed screening process was displayed in Fig. 1.

\section{Statistical analysis}

The data on continuous variables with normal distribution were expressed as mean \pm standard deviation (SD), and median (interquartile range, IQR) was presented for continuous variables with skewed distribution. In the competing risk model, OCSM was regarded as a competing event for LC-SM. First, we computed the cumulative incidence function (CIF) for LC-SM and OCSM. Further subgroup analysis was carried out according to age, gender, race, marital status, tumor laterality, tumor site, histologic grade, TNM stage, and surgery, and corresponding CIF curves were plotted for these variables, respectively. The statistical differences in CIF among subgroups were evaluated by Gray's test [18]. Second, the data set was randomly split into a training cohort (2/3) and a validation cohort (1/3). In total, 1,803 cases served as training cohort were employed for model development, and 902 cases served as validation cohort were used for model validation. Third, variables that were perceived clinically relevant beforehand or considered significant in the univariate analysis $(P<0.1)$ were introduced into a stepwise competing risk regression model. Subsequently, the optimal regression model was fitted when incorporating the predictive variables selected by the stepwise regression procedure. Finally, we calculated the subdistribution hazard ratio (SHR) of the included variables for LC-SM and OCSM based on the multivariate competing risk model, and nomograms on the basis of coefficients from the model were developed. For evaluating the model performance, concordance index (C-index) was utilized to estimate the predictive accuracy (discrimination), and calibration curves (agreement between the observed probability and predicted probability at a certain time point) were constructed to assess the calibration with the aid of R package of "riskRegression" [19]. We also performed a 10-fold cross validation for all data set which was randomly partitioned into 10 equal-sized subsamples [20]. All statistical analyses were carried out employing the $R$ software version 3.5.2. A two-tailed $P<0.05$ was considered statistically significant.

\section{Results}

The baseline characteristics of the whole study cohort were presented in Table 1. In general, a total of 2,705 lung ASC patients were identified in the SEER database. Of these, the median age at diagnosis was 69 years (IQR: 61-76). A 
larger proportion of patients were aged above 65 years $(1,776,65.7 \%)$, male $(1,437,53.1 \%)$, married $(1,590,58.8 \%)$, and white race $(2,248,83.1 \%)$. The majority of tumors were located in the upper lobe $(1,651,61.0 \%)$, and on the right side $(1,564,57.8 \%)$. Most patients were diagnosed with histological grade III (64.7\%), followed by grade II (31.7\%), grade IV (2.3\%), and grade I (1.3\%). The proportion of AJCC stage was $42.2 \%$ for stage I, $12.3 \%$ for stage II, $23.4 \%$ for stage III, and $22.1 \%$ for stage IV. A total of $67.2 \%$ of patients received surgical treatment. 
Table 1

Cumulative incidences of death among patients with ASC.

\begin{tabular}{|c|c|c|c|c|c|c|c|c|}
\hline \multirow[t]{2}{*}{ Variables } & \multirow[t]{2}{*}{$\mathrm{Nt}(\%)$} & \multirow[t]{2}{*}{$\mathrm{Ne}(\%)$} & \multicolumn{4}{|c|}{ LC-SM (\%) } & \multicolumn{2}{|l|}{$\operatorname{OCSM}(\%)$} \\
\hline & & & $\begin{array}{l}\text { 3-year } \\
(95 \% \mathrm{Cl})\end{array}$ & $\begin{array}{l}\text { 5-year } \\
(95 \% \mathrm{Cl})\end{array}$ & $\mathbf{P}$ & $\begin{array}{l}\text { 3-year } \\
(95 \% \mathrm{Cl})\end{array}$ & $\begin{array}{l}\text { 5-year(95\% } \\
\text { Cl) }\end{array}$ & $P$ \\
\hline Total & 2705 & 1897 & $\begin{array}{l}49.6(47.7- \\
51.5)\end{array}$ & $\begin{array}{l}55.8(53.8- \\
57.8)\end{array}$ & & $\begin{array}{l}8.2(7.1- \\
9.2)\end{array}$ & $\begin{array}{l}11.8(10.5- \\
13.1)\end{array}$ & \\
\hline \multicolumn{9}{|l|}{ Age (years) } \\
\hline $\begin{array}{l}\text { median } \\
\text { (IQR) }\end{array}$ & $69(61-76)$ & $70(62-77)$ & & & & & & \\
\hline$<65$ years & $929(34.3)$ & $608(32.1)$ & $\begin{array}{l}49.2(45.9- \\
52.4)\end{array}$ & $\begin{array}{l}55.0(51.6- \\
58.3)\end{array}$ & $\overrightarrow{0.05}$ & $\begin{array}{l}5.1(3.7- \\
6.5)\end{array}$ & $\begin{array}{l}8.0(6.2- \\
9.9)\end{array}$ & $<.001$ \\
\hline$\geq_{65}$ years & 1776(65.7) & 1289(67.9) & $\begin{array}{l}49.9(47.5- \\
52.2)\end{array}$ & $\begin{array}{l}56.3(53.8- \\
58.7)\end{array}$ & & $\begin{array}{l}9.8(8.4- \\
11.2)\end{array}$ & $\begin{array}{l}13.9(12.2- \\
15.6)\end{array}$ & \\
\hline Gender & & & & & 0.002 & & & $\overrightarrow{0.05}$ \\
\hline Female & 1268(46.9) & $858(45.2)$ & $\begin{array}{l}45.8(43.0- \\
48.6)\end{array}$ & $\begin{array}{l}52.4(49.5- \\
55.3)\end{array}$ & & $\begin{array}{l}8.3(6.8- \\
9.9)\end{array}$ & $\begin{array}{l}11.9(10.0- \\
13.8)\end{array}$ & \\
\hline Male & 1437(53.1) & 1039(54.8) & $\begin{array}{l}53.0(50.4- \\
55.6)\end{array}$ & $\begin{array}{l}58.8(56.1- \\
61.5)\end{array}$ & & $\begin{array}{l}8.0(6.6- \\
9.5)\end{array}$ & $\begin{array}{l}11.8(10.0- \\
13.6)\end{array}$ & \\
\hline Race & & & & & 0.008 & & & 0.03 \\
\hline Black & $262(9.7)$ & 195(10.3) & $\begin{array}{l}56.8(50.7- \\
63.0)\end{array}$ & $\begin{array}{l}62.9(56.7- \\
69.1)\end{array}$ & & $\begin{array}{l}7.0(3.9- \\
10.2)\end{array}$ & $\begin{array}{l}12.4(8.0- \\
16.8)\end{array}$ & \\
\hline White & 2248(83.1) & 1571(82.8) & $\begin{array}{l}48.4(46.3- \\
50.5)\end{array}$ & $\begin{array}{l}54.4(52.2- \\
56.6)\end{array}$ & & $\begin{array}{l}8.6(7.4- \\
9.8)\end{array}$ & $\begin{array}{l}12.2(10.8- \\
13.7)\end{array}$ & \\
\hline Other race & 195(7.2) & 131(6.9) & $\begin{array}{l}54.3(46.9- \\
61.7)\end{array}$ & $\begin{array}{l}63.1(55.6- \\
70.6)\end{array}$ & & $\begin{array}{l}4.3(1.4- \\
7.3)\end{array}$ & $\begin{array}{l}6.0(2.3- \\
9.7)\end{array}$ & \\
\hline $\begin{array}{l}\text { Marital } \\
\text { status }\end{array}$ & & & & & 0.02 & & & $\overrightarrow{0.05}$ \\
\hline Married & 1590(58.8) & 1091(57.5) & $\begin{array}{l}48.1(45.5- \\
50.6)\end{array}$ & $\begin{array}{l}54.2(51.6- \\
56.8)\end{array}$ & & $\begin{array}{l}7.9(6.5- \\
9.2)\end{array}$ & $\begin{array}{l}11.3(9.7- \\
13.0)\end{array}$ & \\
\hline Unmarried & 1115(41.2) & $806(42.5)$ & $\begin{array}{l}51.9(48.9- \\
54.9)\end{array}$ & $\begin{array}{l}58.1(55.1- \\
61.2)\end{array}$ & & $\begin{array}{l}8.6(6.9- \\
10.3)\end{array}$ & $\begin{array}{l}12.6(10.5- \\
14.7)\end{array}$ & \\
\hline Tumor site & & & & & $\begin{array}{l}< \\
0.001\end{array}$ & & & $\overrightarrow{0.05}$ \\
\hline Upper lobe & 1651(61.0) & 1130(59.6) & $\begin{array}{l}47.8(45.3- \\
50.3)\end{array}$ & $\begin{array}{l}53.7(51.2- \\
56.3)\end{array}$ & & $\begin{array}{l}8(6.7- \\
9.4)\end{array}$ & $\begin{array}{l}12(10.3- \\
13.7)\end{array}$ & \\
\hline Middle lobe & $113(4.2)$ & $79(4.2)$ & $\begin{array}{l}42.4(33.1- \\
51.7)\end{array}$ & $\begin{array}{l}53.8(43.7- \\
63.8)\end{array}$ & & $\begin{array}{l}11.2(5.1- \\
17.2)\end{array}$ & $\begin{array}{l}18.6(10.6- \\
26.6)\end{array}$ & \\
\hline
\end{tabular}




\begin{tabular}{|c|c|c|c|c|c|c|c|c|}
\hline \multirow[t]{2}{*}{ Variables } & \multirow[t]{2}{*}{$\mathrm{Nt}(\%)$} & \multirow[t]{2}{*}{$\mathrm{Ne}(\%)$} & \multicolumn{3}{|c|}{ LC-SM (\%) } & \multicolumn{3}{|c|}{$\operatorname{OCSM}(\%)$} \\
\hline & & & $\begin{array}{l}\text { 3-year } \\
(95 \% \text { Cl) }\end{array}$ & $\begin{array}{l}\text { 5-year } \\
(95 \% \mathrm{Cl})\end{array}$ & $P$ & $\begin{array}{l}\text { 3-year } \\
(95 \% \mathrm{Cl})\end{array}$ & $\begin{array}{l}\text { 5-year(95\% } \\
\mathrm{Cl})\end{array}$ & $P$ \\
\hline Lower lobe & $838(31.0)$ & $605(31.9)$ & $\begin{array}{l}52.3(48.8- \\
55.7)\end{array}$ & $\begin{array}{l}58.2(54.7- \\
61.7)\end{array}$ & & $\begin{array}{l}8.5(6.5- \\
10.4)\end{array}$ & $\begin{array}{l}11.4(9.1- \\
13.7)\end{array}$ & \\
\hline $\begin{array}{l}\text { Main } \\
\text { bronchus }\end{array}$ & $58(2.1)$ & $53(2.8)$ & $\begin{array}{l}82(71.6- \\
92.5)\end{array}$ & $\begin{array}{l}\text { 86.8(76.9- } \\
96.7)\end{array}$ & & $\begin{array}{l}5.2(0- \\
11)\end{array}$ & $\begin{array}{l}5.2(0- \\
11.0)\end{array}$ & \\
\hline $\begin{array}{l}\text { Overlapping } \\
\text { lesion }\end{array}$ & $45(1.7)$ & $30(1.6)$ & $\begin{array}{l}42.8(28- \\
57.6)\end{array}$ & $\begin{array}{l}52.8(37.5- \\
68.0)\end{array}$ & & $\begin{array}{l}4.6(0- \\
11)\end{array}$ & $\begin{array}{l}7.1(0- \\
15.0)\end{array}$ & \\
\hline $\begin{array}{l}\text { Tumor } \\
\text { laterality }\end{array}$ & & & & & $\begin{array}{l}> \\
0.05\end{array}$ & & & $\overrightarrow{0} .05$ \\
\hline Right & 1564(57.8) & 1105(58.2) & $\begin{array}{l}48.7(46.2- \\
51.2)\end{array}$ & $\begin{array}{l}55.2(52.6- \\
57.8)\end{array}$ & & $\begin{array}{l}8.6(7.2- \\
10)\end{array}$ & $\begin{array}{l}12.9(11.1- \\
14.7)\end{array}$ & \\
\hline Left & $1135(42.0)$ & $787(41.5)$ & $\begin{array}{l}50.8(47.9- \\
53.8)\end{array}$ & $\begin{array}{l}56.4(53.4- \\
59.4)\end{array}$ & & $7.6(6-9.2)$ & $\begin{array}{l}10.4(8.5- \\
12.3)\end{array}$ & \\
\hline Bilateral & $6(0.2)$ & $5(0.3)$ & $\begin{array}{l}\text { 66.7(22.2- } \\
111.2)\end{array}$ & $\begin{array}{l}83.3(43.8- \\
100)\end{array}$ & & $0(0-0)$ & $0(0-0)$ & \\
\hline Grade & & & & & $\begin{array}{l}< \\
0.001\end{array}$ & & & $<001$ \\
\hline Grade I & $35(1.3)$ & $21(1.1)$ & $\begin{array}{l}21.1(6.8- \\
35.3)\end{array}$ & $\begin{array}{l}21.1(6.8- \\
35.3)\end{array}$ & & $\begin{array}{l}21.5(7- \\
36)\end{array}$ & $\begin{array}{l}25.6(9.6- \\
41.5)\end{array}$ & \\
\hline Grade II & $857(31.7)$ & $529(27.9)$ & $\begin{array}{l}38.2(34.8- \\
41.5)\end{array}$ & $\begin{array}{l}45.9(42.4- \\
49.5)\end{array}$ & & $\begin{array}{l}7.5(5.7- \\
9.3)\end{array}$ & $\begin{array}{l}12.8(10.4- \\
15.3)\end{array}$ & \\
\hline Grade III & 1751(64.7) & $1294(68.2)$ & $\begin{array}{l}55.4(53.1- \\
57.8)\end{array}$ & $\begin{array}{l}\text { 61.1(58.7- } \\
63.5)\end{array}$ & & $\begin{array}{l}8.3(6.9- \\
9.6)\end{array}$ & $\begin{array}{l}11.1(9.5- \\
12.6)\end{array}$ & \\
\hline Grade IV & $62(2.3)$ & $53(2.8)$ & $\begin{array}{l}60.1(47.7- \\
72.6)\end{array}$ & $\begin{array}{l}62.1(49.6- \\
74.6)\end{array}$ & & $\begin{array}{l}8.2(1.2- \\
15.2)\end{array}$ & $\begin{array}{l}12.2(3.5- \\
20.8)\end{array}$ & \\
\hline T stage & & & & & $<.001$ & & & $<0.001$ \\
\hline T1 & $736(27.2)$ & $402(21.2)$ & $\begin{array}{l}26.8(23.5- \\
30.1)\end{array}$ & $\begin{array}{l}32.8(29.2- \\
36.4)\end{array}$ & & $\begin{array}{l}9.4(7.2- \\
11.6)\end{array}$ & $\begin{array}{l}16.3(13.3- \\
19.2)\end{array}$ & \\
\hline T2 & $1213(44.8)$ & $839(44.2)$ & $\begin{array}{l}47.2(44.4- \\
50.1)\end{array}$ & $\begin{array}{l}54.2(51.3- \\
57.2)\end{array}$ & & $\begin{array}{l}9(7.4- \\
10.7)\end{array}$ & $\begin{array}{l}12.1(10.2- \\
14.0)\end{array}$ & \\
\hline T3 & $211(7.8)$ & $174(9.2)$ & $\begin{array}{l}68.9(62.5- \\
75.3)\end{array}$ & $\begin{array}{l}74.6(68.4- \\
80.9)\end{array}$ & & $\begin{array}{l}6.6(3.1- \\
10.1)\end{array}$ & $\begin{array}{l}9.9(5.5- \\
14.4)\end{array}$ & \\
\hline T4 & $545(20.1)$ & $482(25.4)$ & $\begin{array}{l}78.2(74.6- \\
81.7)\end{array}$ & $\begin{array}{l}83.1(79.7- \\
86.5)\end{array}$ & & $\begin{array}{l}5.2(3.3- \\
7.1)\end{array}$ & $\begin{array}{l}6.1(4.0- \\
8.2)\end{array}$ & \\
\hline $\mathrm{N}$ stage & & & & & $\begin{array}{l}< \\
0.001\end{array}$ & & & $\begin{array}{l}< \\
0.001\end{array}$ \\
\hline
\end{tabular}

Abbreviations: $\mathrm{N}_{\mathrm{t}}$ : total number; $\mathrm{N}_{\mathrm{e}}$ : number of death events; ASC: adenosquamous carcinoma; Cl: confidence interval; LC-SM: lung cancer-specific mortality; OCSM: other cause-specific morality; AJCC: American Joint Committee on Cancer; IQR: interquartile range. 


\begin{tabular}{|c|c|c|c|c|c|c|c|c|}
\hline \multirow[t]{2}{*}{ Variables } & \multirow[t]{2}{*}{$\mathrm{Nt}(\%)$} & \multirow[t]{2}{*}{$\mathrm{Ne}(\%)$} & \multicolumn{3}{|c|}{ LC-SM (\%) } & \multicolumn{3}{|c|}{ OCsM (\%) } \\
\hline & & & $\begin{array}{l}\text { 3-year } \\
(95 \% \mathrm{Cl})\end{array}$ & $\begin{array}{l}\text { 5-year } \\
(95 \% \mathrm{Cl})\end{array}$ & $\mathbf{P}$ & $\begin{array}{l}\text { 3-year } \\
(95 \% \mathrm{Cl})\end{array}$ & $\begin{array}{l}5-\operatorname{year}(95 \% \\
\mathrm{Cl})\end{array}$ & $P$ \\
\hline NO & $1475(54.5)$ & $892(47.0)$ & $\begin{array}{l}33.8(31.4- \\
36.3)\end{array}$ & $\begin{array}{l}40.6(38.0- \\
43.2)\end{array}$ & & $\begin{array}{l}9.6(8- \\
11.1)\end{array}$ & $\begin{array}{l}14.9(12.9- \\
16.9)\end{array}$ & \\
\hline $\mathrm{N} 1$ & $362(13.4)$ & $245(12.9)$ & $\begin{array}{l}51.5(46.2- \\
56.7)\end{array}$ & $\begin{array}{l}58.6(53.2- \\
64.0)\end{array}$ & & $5.6(3.1-8)$ & $\begin{array}{l}8.3(5.2- \\
11.4)\end{array}$ & \\
\hline N2 & $711(26.3)$ & $617(32.5)$ & $\begin{array}{l}73.8(70.5- \\
77.1)\end{array}$ & $\begin{array}{l}79.2(76.0- \\
82.3)\end{array}$ & & $\begin{array}{l}7(5.1- \\
8.9)\end{array}$ & $\begin{array}{l}8.3(6.2- \\
10.4)\end{array}$ & \\
\hline N3 & 157(5.8) & $143(7.5)$ & $\begin{array}{l}85.1(79.2- \\
91.0)\end{array}$ & $\begin{array}{l}\text { 87.3(81.5- } \\
93.2)\end{array}$ & & $\begin{array}{l}6.6(2.6- \\
10.5)\end{array}$ & $\begin{array}{l}6.6(2.6- \\
10.5)\end{array}$ & \\
\hline M stage & & & & & $\begin{array}{l}< \\
0.001\end{array}$ & & & $\begin{array}{l}<.001 \\
0.00\end{array}$ \\
\hline MO & 2108(77.9) & 1344(70.8) & $\begin{array}{l}39.8(37.7- \\
42)\end{array}$ & $\begin{array}{l}46.9(44.7- \\
49.2)\end{array}$ & & $\begin{array}{l}8.9(7.6- \\
10.1)\end{array}$ & $\begin{array}{l}13.3(11.8- \\
14.9)\end{array}$ & \\
\hline M1 & $597(22.1)$ & $553(29.2)$ & $\begin{array}{l}84.2(81.2- \\
87.1)\end{array}$ & $\begin{array}{l}87.1(84.2- \\
89.9)\end{array}$ & & $\begin{array}{l}5.8(3.9- \\
7.7)\end{array}$ & $\begin{array}{l}6.5(4.5- \\
8.6)\end{array}$ & \\
\hline AJCC Stage & & & & & $<0.001$ & & & $\begin{array}{l}< \\
0.001\end{array}$ \\
\hline I & $\begin{array}{l}1142 \\
(42.2)\end{array}$ & $624(32.9)$ & $\begin{array}{l}23.9(21.4- \\
26.4)\end{array}$ & $\begin{array}{l}31.1(28.3- \\
34.0)\end{array}$ & & $\begin{array}{l}11.2(9.3- \\
13.1)\end{array}$ & $\begin{array}{l}17.2(14.8- \\
19.6)\end{array}$ & \\
\hline ॥ & $332(12.3)$ & $213(11.2)$ & $\begin{array}{l}47.1(41.6- \\
52.6)\end{array}$ & $\begin{array}{l}52.7(47.1- \\
58.4)\end{array}$ & & $\begin{array}{l}5.7(3.1- \\
8.3)\end{array}$ & $\begin{array}{l}9.4(6.0- \\
12.8)\end{array}$ & \\
\hline III & $634(23.4)$ & $507(26.7)$ & $\begin{array}{l}64.9(61.1- \\
68.8)\end{array}$ & $\begin{array}{l}72.8(69.1- \\
76.5)\end{array}$ & & $\begin{array}{l}6.3(4.3- \\
8.2)\end{array}$ & $\begin{array}{l}8.4(6.0- \\
10.7)\end{array}$ & \\
\hline IV & $597(22.1)$ & $553(29.2)$ & $\begin{array}{l}84.2(81.2- \\
87.1)\end{array}$ & $\begin{array}{l}87.1(84.2- \\
89.9)\end{array}$ & & $\begin{array}{l}5.8(3.9- \\
7.7)\end{array}$ & $\begin{array}{l}6.5(4.5- \\
8.6)\end{array}$ & \\
\hline Surgery & & & & & $<.001$ & & & $\begin{array}{l}<.001 \\
0 .\end{array}$ \\
\hline No & $886(32.8)$ & $806(42.5)$ & $\begin{array}{l}82.1(79.5- \\
84.7)\end{array}$ & $\begin{array}{l}85.9(83.4- \\
88.5)\end{array}$ & & $\begin{array}{l}7.9(6.1- \\
9.7)\end{array}$ & $\begin{array}{l}8.8(6.8- \\
10.8)\end{array}$ & \\
\hline Yes & $1819(67.2)$ & 1091(57.5) & $\begin{array}{l}34.1(31.8- \\
36.3)\end{array}$ & $\begin{array}{l}41.7(39.3- \\
44.0)\end{array}$ & & $8.3(7-9.6)$ & $\begin{array}{l}13.3(11.6- \\
15.0)\end{array}$ & \\
\hline $\begin{array}{l}\text { Abbreviations } \\
\text { interval; LC-S } \\
\text { Committee o }\end{array}$ & $\begin{array}{l}\mathrm{V}_{\mathrm{t}} \text { : total num } \\
\text { lung cancer } \\
\text { Cancer; IQR: i }\end{array}$ & $\begin{array}{l}\text { r; } \mathrm{N}_{\mathrm{e}} \text { : numbe } \\
\text { pecific morta } \\
\text { erquartile rar }\end{array}$ & $\begin{array}{l}\text { of death ever } \\
\text { y; OCSM: oth }\end{array}$ & $\begin{array}{l}\text {; ASC: adenc } \\
\text { cause-speci }\end{array}$ & $\begin{array}{l}\text { squamou } \\
\text { c moralit }\end{array}$ & $\begin{array}{l}\text { s carcinoma, } \\
\text { y; AJCC: Am }\end{array}$ & $\begin{array}{l}\text { Cl: confidence } \\
\text { rican Joint }\end{array}$ & \\
\hline
\end{tabular}

The median follow-up of the whole study cohort was 21 months (IQR: 8-52). In total, 1,895 (70.1\%) patients died throughout the whole follow-up period, of whom 1,535 (81.0\%) died owing to lung cancer and 362 (19.0\%) died due to non-lung cancer causes. The 3-year and 5-year cumulative incidences of LC-SM and OCSM by different clinicopathological characteristics were displayed in Table 1, and the corresponding CIF curves were presented in Fig. 2. Overall, the 3-year and 5-year LC-SM were $49.6 \%(\mathrm{Cl}: 47.7 \%-51.5 \%)$ and 55.8\% (Cl: 53.8\%-57.8\%), respectively, while the 3-year and 5-year OCSM were 8.2\% (Cl: 7.1\%-9.2\%) and 11.8\% (Cl: 10.5\%-13.1\%), respectively. In univariate analysis, male, unmarried status, black race, main bronchus, advanced TNM stage, advanced histological grade, and surgical 
treatment were related to significantly higher incidences of LC-SM, whereas there were no significant differences for age and tumor laterality. In the meantime, there were significantly higher incidences of OCSM for patients aged $\geq 65$ years, diagnosed with histological grade I or earlier TNM stage, and receiving surgery.

In multivariate analysis, age, gender, surgery, T stage, $\mathrm{N}$ stage, and $\mathrm{M}$ stage were independent predictive factors for LCSM, in contrast with merely age and surgery acting as independent predictors for OCSM (Table 2). Furthermore, increasing age was associated with an increased probability of LCSM. Male was related to a significantly higher likelihood of LCSM (1.26, Cl: 1.10-1.43), while surgery was related to a significantly lower likelihood of LC-SM (0.45, Cl: 0.37-0.53). When compared with patients with T1, advanced T-stage patients had a higher probability to face LCSM, with SHR of 1.44 (1.21-1.72), 2.24 (1.72-2.92), and 1.99 (1.59-2.49) for T2, T3, and T4, respectively. Similar phenomenon was observed among advanced N-stage patients, with SHR of 1.52(1.26-1.84), 1.57(1.32-1.87), and 1.51(1.12-2.03) for N1, N2, and N3, respectively, in contrast with N0. For OCSM, patients with older age and receiving surgery were at a higher risk. 
Table 2

Multivariate competing risk model for LC-SM and OCSM in patients with lung ASC.

\begin{tabular}{|c|c|c|c|c|c|c|}
\hline \multirow[t]{2}{*}{ Characteristics } & \multicolumn{3}{|l|}{ LC-SM } & \multicolumn{3}{|l|}{ OCSM } \\
\hline & Coefficient & SHR (95\% Cl) & $\mathbf{P}$ & Coefficient & SHR (95\% Cl) & $\mathbf{P}$ \\
\hline Age & 0.009 & $1.01(1.00-1.02)$ & 0.008 & 0.035 & $1.04(1.02-1.05)$ & $<0.001$ \\
\hline \multicolumn{7}{|l|}{ Sex } \\
\hline Female & Reference & Reference & & & & \\
\hline Male & 0.228 & $1.26(1.10-1.43)$ & $<0.001$ & & & \\
\hline \multicolumn{7}{|l|}{ Surgery } \\
\hline No & Reference & Reference & & Reference & Reference & \\
\hline Yes & -0.810 & $0.45(0.37-0.53)$ & $<0.001$ & 0.695 & $2.00(1.46-2.75)$ & $<0.001$ \\
\hline \multicolumn{7}{|l|}{ T stage } \\
\hline $\mathrm{T} 1$ & Reference & Reference & & & & \\
\hline T2 & 0.363 & $1.44(1.21-1.72)$ & $<0.001$ & & & \\
\hline T3 & 0.805 & $2.24(1.72-2.92)$ & $<0.001$ & & & \\
\hline T4 & 0.688 & $1.99(1.59-2.49)$ & $<0.001$ & & & \\
\hline \multicolumn{7}{|l|}{$\mathrm{N}$ stage } \\
\hline NO & Reference & Reference & & & & \\
\hline N1 & 0.419 & $1.52(1.26-1.84)$ & $<0.001$ & & & \\
\hline N2 & 0.454 & $1.57(1.32-1.87)$ & $<0.001$ & & & \\
\hline N3 & 0.409 & $1.51(1.12-2.03)$ & 0.007 & & & \\
\hline \multicolumn{7}{|l|}{ M stage } \\
\hline Mo & Reference & Reference & & & & \\
\hline M1 & 0.506 & $1.66(1.38-2.00)$ & $<0.001$ & & & \\
\hline
\end{tabular}

The nomograms on the basis of the competing risk models were developed to calculate the 3-year and 5-year cumulative death probabilities (Fig. 3). For each patient, locate the values of different variables on the corresponding variable rows and draw vertical lines pointing to the "Points" row to get corresponding scores. For instance, for a male patient, by drawing a vertical line straight up to the "Point" row, we would obtain approximately 28 points. Similar processes were performed to other variables. By adding up these scores, a total score was obtained, and can be located on the "Total Points" row. Subsequently, a vertical line was drawn straight down to acquire the 3-year or 5-year cumulative death probabilities. For example, if the total score was 100, the corresponding 3-year and 5-year probabilities of LC-SM would be approximately $30 \%$ and $36 \%$, respectively. 
The calibration curves accompanied with C-indexes were displayed in Fig. 4. As shown in Fig. 4, if the calibration curves were close to the 45-degree diagonal line, it denoted the developed nomograms were well calibrated (a good agreement between the observed mortality probability and predicted mortality probability). Additionally, the 3-year and 5-year Cindexes for the nomograms predicting probabilities of LC-SM were $0.83(\mathrm{Cl}, 0.78-0.87)$ and $0.82(\mathrm{Cl}, 0.73-0.90)$ for training cohort, and $0.79(\mathrm{Cl}, 0.75-0.84)$ and $0.79(\mathrm{Cl}, 0.71-0.88)$ for validation cohort, respectively, which indicated a superb model discrimination. Besides, when it comes to the nomograms predicting probabilities of OCSM, the 3-year and 5-year C-indexes were $0.60(\mathrm{Cl}, 0.55-0.65)$ and $0.62(\mathrm{Cl}, 0.57-0.67)$ for training cohort, as well as $0.58(\mathrm{Cl}, 0.50-$ $0.66)$ and $0.63(\mathrm{Cl}, 0.56-0.70)$ for validation cohort, respectively. The 10-fold cross validation C-indexes were shown in Table 3. The adjusted 3-year and 5-year C-indexes were $0.81(\mathrm{Cl}, 0.80-0.83)$ and $0.81(\mathrm{Cl}, 0.80-0.83)$ for predicting probabilities of LC-SM, and $0.60(\mathrm{Cl}, 0.56-0.63)$ and $0.63(\mathrm{Cl}, 0.58-0.65)$ for predicting probabilities of OCSM.

Table 3

C-indexes of the predictive model for patients with ASC.

\begin{tabular}{|c|c|c|c|c|}
\hline \multirow[t]{2}{*}{ Cohort } & \multicolumn{2}{|l|}{ C-indexes } & \multicolumn{2}{|c|}{ Adjusted C-indexes } \\
\hline & 3-year & 5-year & 3-year & 5-year \\
\hline \multicolumn{5}{|c|}{ Training cohort } \\
\hline LC-SM & $\begin{array}{l}0.83(\mathrm{Cl}, 0.78- \\
0.87)\end{array}$ & $\begin{array}{l}0.82(\mathrm{Cl}, 0.73- \\
0.90)\end{array}$ & & \\
\hline OCSM & $\begin{array}{l}0.60(\mathrm{Cl}, 0.55- \\
0.65)\end{array}$ & $\begin{array}{l}0.62(\mathrm{Cl}, 0.57- \\
0.67)\end{array}$ & & \\
\hline \multicolumn{5}{|c|}{$\begin{array}{l}\text { Validation } \\
\text { cohort }\end{array}$} \\
\hline LC-SM & $\begin{array}{l}0.79(\mathrm{Cl}, 0.75- \\
0.84)\end{array}$ & $\begin{array}{l}0.79(\mathrm{Cl}, 0.71- \\
0.88)\end{array}$ & & \\
\hline ocsM & $\begin{array}{l}0.58(\mathrm{Cl}, 0.50- \\
0.66)\end{array}$ & $\begin{array}{l}0.63(\mathrm{Cl}, 0.56- \\
0.70)\end{array}$ & & \\
\hline \multicolumn{5}{|c|}{ Overall cohort } \\
\hline LC-SM & & & $\begin{array}{l}0.81(\mathrm{Cl}, 0.80- \\
0.83)\end{array}$ & $\begin{array}{l}0.81(\mathrm{Cl}, 0.80- \\
0.83)\end{array}$ \\
\hline OCSM & & & $\begin{array}{l}0.60(\mathrm{Cl}, 0.56- \\
0.63)\end{array}$ & $\begin{array}{l}0.63(\mathrm{Cl}, 0.58- \\
0.65)\end{array}$ \\
\hline
\end{tabular}

\section{Discussion}

In the present study, a competing risk analysis was performed to investigate the predictive factors for LC-SM and OCSM among patients with primary lung ASC from the SEER database. Of the total 2,705 patients, 1,535 (81.0\%) died from lung cancer and 362 (19.0\%) died from non-lung cancer causes. The 5-year CIFs for LC-SM and OCSM were $55.8 \%$ and $11.8 \%$, respectively. We constructed nomograms to function as a simple and useful clinical tool to predict the individual probabilities of LC-SM and OCSM for lung ASC patients.

With regard to LC-SM, T, N, and M stage were significant independent predictive factors, unanimous with the eighth edition of AJCC NSCLC staging system [21]. Besides, we also identified other important predictors, such as age, sex, and surgery, which have been incorporated into our nomograms. These predictors of an increased LC-SM, including advanced age, male sex, and surgery, have been proved in other studies [22, 23]. For example, a recent retrospective 
study investigating NSCLC based on the SEER database found that advanced age and male sex were related to decreased lung cancer-specific survival, and any form of surgical resection conferred a decreased risk of lung cancerspecific mortality [22]. H Zhou et al analyzed data from patients with radically resected stage I NSCLC in the SEER database and discovered that advanced age and male sex were correlated with a higher risk of cause-specific death [23]. As the AJCC staging system does not include important risk factors (including age, sex and surgery), the nomograms we developed were more discriminative and were capable of providing a more accurate prognostic prediction for individual patients.

In this study, we found increasing age and surgery were related to an elevated probability of OCSM, indicating the clinical importance of evaluating OCSM as a competing risk event for elderly patients who were eligible for surgical treatment. Similar to our findings, several studies have found advanced age was an independent unfavorable prognostic factor for lung ASC patients [3, 7, 22, 24]. However, they mainly focused on the overall survival (OS) rather than OCSM, which might bias their findings, resulting in either overtreatment or undertreatment. Wei $S$ et al found surgery (except for lobectomy or bilobectomy) conferred an increased risk of OCSM when compared with no surgery, despite the main study population being NSCLC rather than ASC. Additionally, in contrast with younger patients, elderly patients were more exposed to severe comorbidities [25, 26], and comorbidities have been demonstrated as a strong predictor of OCSM in various cancers [9-11]. Therefore, for elderly patients with severe comorbidities, surgery might not be a favorable treatment means, allowing for the high risk of OCSM. In general, it is essential for clinicians to evaluate the overall health status and surgery tolerance degree for elderly patients, and provide a better basic life support for them.

For the sake of patient counseling and clinical decision making, it is imperative to evaluate prognosis according to individual risk profiles. With the aid of prognostic nomograms, it is more expedient for clinicians to devise treatment protocols and follow-up strategies. Notably, competing risk nomograms have been developed for various cancers, such as nasopharyngeal carcinoma, breast cancer, gastrointestinal stromal tumors and melanoma [27-30]. However, as far as we know, this is the first report constructing competing risk nomograms by virtue of the proportional subdistribution hazard model to predict the individual probabilities of LC-SM and OCSM for lung ASC patients.

The major strengths of the present study are that it had a large enough sample size and adopted a competing risk model to perform survival analysis. In general, the SEER database, covering about $27.8 \%$ of the US population, offers a sufficiently large sample to investigate predictive factors and further develop a model-based prognostic nomogram. Moreover, findings derived from the analysis based on the population-based database were more generalizable and representative than those from single-center studies [31]. Meanwhile, competing risk model fully take into consideration the competing events, which renders the results more unbiased. Notably, the variables presented in the nomogram can be easily collected from the routine medical records, which makes it more expedient for clinicians to predict the cumulative death probabilities for lung ASC patients.

Undoubtedly, there are several limitations in this study. First, some known prognostic variables, such as cigarette smoking, chemotherapy, radiation therapy, and comorbidity, were not incorporated into the model. Thus, the nomogram is only functioned as a reference tool for clinicians to make clinical decisions. Further study is warranted to incorporate these variables into the future research. Second, as the whole study population was from the US, the findings of the present study may not be generalizable to other countries. Finally, although our model exhibits an excellent performance in predicting the probabilities of LC-SM (with C-indexes fluctuating around 0.8 ), the discrimination for predicting the probabilities of OCSM was merely within the range of 0.6 to 0.65 . Therefore, it is essential to identify more risk factors for predicting OCSM. In addition, external validation cohort including other patients is still necessary to demonstrate the model accuracy. 


\section{Conclusions}

In conclusion, this is the first study using a competing risk model to evaluate the cumulative incidence of LC-SM and OCSM for patients with lung ASC. We further developed a competing risk nomogram, and the nomogram displayed an excellent discrimination and calibration. With the aid of this individualized predictive tool, it is more expedient for clinicians to devise appropriate treatment protocols and follow-up schedules.

\section{Abbreviations}

ASC: Adenosquamous carcinoma; ADC: Adenocarcinoma; SCC: Squamous cell carcinoma; OCSM: Other cause-specific mortality; NSCLC: Non-small-cell Lung Cancer; LC-SM: Lung cancer-specific mortality; SEER: Surveillance, Epidemiology, and End Results; ICD-0-3: International Classification of Diseases for Oncology, Third Edition; AJCC: American Joint Committee on Cancer; SD: Standard deviation; IQR: Interquartile range; CIF: Cumulative incidence function; C-index: Concordance index.

\section{Declarations}

\section{Ethics approval and consent to participate}

As all the data of this study were derived from the SEER database, institutional review board approval and consent to participate were not demanded.

\section{Consent for publication}

Not applicable.

\section{Availability of data and materials}

All the data of this study were derived from the SEER database, which was available from: www.seer.cancer.gov.

\section{Competing interests}

PR has received speaker fee from Medtronic. The other authors did not report conflicts of interest.

\section{Funding}

This work was supported by The National Key Research and Development Program of China (No. 2017YFC0113500), Zhejiang Province Major Science and Technology Special Project (2014C03032), Zhejiang Provincial Key Discipline of Traditional Chinese Medicine (2017-XK-A33).

\section{Author' Contributions}

XW, WY, and JH designed and wrote the manuscript; XW, PR, HS, YW, WL, and JH participated in literature search, data acquisition, data analysis, or data interpretation; XW, WY, and $\mathrm{JH}$ contributed to the revision of manuscript. All authors approved the final version to be published.

\section{Acknowledgements}

Not applicable. 


\section{References}

1. Takamori S, Noguchi M, Morinaga S, Goya T, Tsugane S, Kakegawa T, Shimosato Y: Clinicopathologic characteristics of adenosquamous carcinoma of the lung. Cancer 1991, 67(3):649-654.

2. Shimoji M, Nakajima T, Yamatani C, Yamamoto M, Saishou S, Isaka M, Maniwa T, Ode Y, Nakagawa K, Okumura T et al: A clinicopathological and immunohistological re-evaluation of adenosquamous carcinoma of the lung. Pathol Int 2011, 61(12):717-722.

3. Mordant P, Grand B, Cazes A, Foucault C, Dujon A, Le Pimpec Barthes F, Riquet M: Adenosquamous carcinoma of the lung: surgical management, pathologic characteristics, and prognostic implications. Ann Thorac Surg 2013, 95(4):1189-1195.

4. Zhu L, Jiang L, Yang J, Gu W, He J: Clinical characteristics and prognosis of patients with lung adenosquamous carcinoma after surgical resection: results from two institutes. J Thorac Dis 2018, 10(4):2397-2402.

5. Brambilla E, Travis WD, Corrin B, Shimosato Y, Brambilla E: Adenosquamous carcinoma. WHO Histological Classification of Tumours Histological Typing of Lung and Pleural Tumours, 3rd edn Berlin: Springer-Verlag 1999:51-52.

6. Cooke DT, Nguyen DV, Yang Y, Chen SL, Yu C, Calhoun RF: Survival comparison of adenosquamous, squamous cell, and adenocarcinoma of the lung after lobectomy. Ann Thorac Surg 2010, 90(3):943-948.

7. Maeda H, Matsumura A, Kawabata T, Suito T, Kawashima O, Watanabe T, Okabayashi K, Kubota I, Japan National Hospital Organization Study Group for Lung C: Adenosquamous carcinoma of the lung: surgical results as compared with squamous cell and adenocarcinoma cases. Eur J Cardiothorac Surg 2012, 41(2):357-361.

8. Janssen-Heijnen ML, Houterman S, Lemmens VE, Louwman MW, Maas HA, Coebergh JW: Prognostic impact of increasing age and co-morbidity in cancer patients: a population-based approach. Crit Rev Oncol Hematol 2005, 55(3):231-240.

9. Vaisanen JA, Alho OP, Koivunen PT, Laara E: Cause-specific mortality in patients with head and neck cancer: Longterm follow-up of a population-based cohort from 1986 to 2012 accounting for competing risks. Oral Oncol 2018, 79:20-26.

10. Whitney CA, Howard LE, Freedland SJ, DeHoedt AM, Amling CL, Aronson WJ, Cooperberg MR, Kane CJ, Terris MK, Daskivich TJ: Impact of age, comorbidity, and PSA doubling time on long-term competing risks for mortality among men with non-metastatic castration-resistant prostate cancer. Prostate Cancer Prostatic Dis 2018, 22(2):252-260.

11. Larcher A, Trudeau V, Dell'Oglio P, Tian Z, Boehm K, Fossati N, Capitanio U, Briganti A, Montorsi F, Karakiewicz P: Prediction of Competing Mortality for Decision-making Between Surgery or Observation in Elderly Patients With T1 Kidney Cancer. Urology 2017, 102:130-137.

12. M. Pintilie. Competing risks: a practical perspective, John Wiley \& Sons Ltd, 2006.

13. Carmona R, Zakeri K, Green G, Hwang L, Gulaya S, Xu B, Verma R, Williamson CW, Triplett DP, Rose BS et al: Improved Method to Stratify Elderly Patients With Cancer at Risk for Competing Events. J Clin Oncol 2016, 34(11):1270-1277.

14. Bianco FJ, Jr.: Nomograms and medicine. Eur Urol 2006, 50(5):884-886.

15. Zeng Y, Mayne N, Yang CJ, D'Amico TA, Ng CSH, Liu CC, Petersen RH, Rocco G, Brunelli A, Liu J et al: A Nomogram for Predicting Cancer-Specific Survival of TNM 8th Edition Stage I Non-small-cell Lung Cancer. Ann Surg Oncol 2019, 26(7):2053-2062.

16. Xiao Z, Yan Y, Zhou Q, Liu H, Huang P, Zhou Q, Lai C, Zhang J, Wang J, Mao K: Development and external validation of prognostic nomograms in hepatocellular carcinoma patients: a population based study. Cancer Manag Res 2019, 11:2691-2708.

Page 13/18 
17. Gross JP, Whelan TJ, Parulekar WR, Chen BE, Rademaker AW, Helenowski IB, Donnelly ED, Strauss JB: Development and validation of a nomogram to predict lymphedema following axillary surgery and radiotherapy in women with breast cancer from the NCIC CTG MA.20 randomized trial. Int J Radiat Oncol Biol Phys 2019, 105(1):165-173.

18. Gray RJ: A class of K-sample tests for comparing the cumulative incidence of a competing risk. Ann Stat 1988, 16(3):1141-1154.

19. T. A. Gerds. riskRegression: Risk Regression Models and Prediction Scores for Survival Analysis with Competing Risks. R package version 2.9.0. https://cran.r-project.org/web/packages/riskRegression/index.html, 2019 Accessed 27 November 2019.

20. Lee M, Cronin KA, Gail MH, Feuer EJ: Predicting the absolute risk of dying from colorectal cancer and from other causes using population-based cancer registry data. Stat Med 2012, 31(5):489-500.

21. National Comprehensive Cancer Network. NCCN clinical practice guidelines in oncology-version V.4.2019 (nonsmall-cell lung cancer). https://www.nccn.org/professionals/physician_gls/pdf/nscl.pdf, 2019 Accessed 27 November 2019.

22. Wei S, Tian J, Song X, Wu B, Liu L: Causes of death and competing risk analysis of the associated factors for nonsmall cell lung cancer using the Surveillance, Epidemiology, and End Results database. J Cancer Res Clin Oncol 2018, 144(1):145-155.

23. Zhou H, Zhang Y, Qiu Z, Chen G, Hong S, Chen X, Zhang Z, Huang Y, Zhang L: Nomogram to Predict Cause-Specific Mortality in Patients With Surgically Resected Stage I Non-Small-Cell Lung Cancer: A Competing Risk Analysis. Clin Lung Cancer 2018, 19(2):e195-e203.

24. Nakagawa K, Yasumitu T, Fukuhara K, Shiono H, Kikui M: Poor prognosis after lung resection for patients with adenosquamous carcinoma of the lung. Ann Thorac Surg 2003, 75(6):1740-1744.

25. Barnett K, Mercer SW, Norbury M, Watt G, Wyke S, Guthrie B: Epidemiology of multimorbidity and implications for health care, research, and medical education: a cross-sectional study. Lancet 2012, 380(9836):37-43.

26. Marengoni A, Angleman S, Melis R, Mangialasche F, Karp A, Garmen A, Meinow B, Fratiglioni L: Aging with multimorbidity: a systematic review of the literature. Ageing Res Rev 2011, 10(4):430-439.

27. Huang XD, Zhou GQ, Lv JW, Zhou HQ, Zhong CW, Wu CF, Zheng ZQ, He XJ, Peng L, Ma J et al: Competing risk nomograms for nasopharyngeal carcinoma in the intensity-modulated radiotherapy era: A big-data, intelligence platform-based analysis. Radiother Oncol 2018, 129(2):389-395.

28. Sun W, Cheng M, Zhou H, Huang W, Qiu Z: Nomogram Predicting Cause-Specific Mortality in Nonmetastatic Male Breast Cancer: A Competing Risk Analysis. J Cancer 2019, 10(3):583-593.

29. Song W, Lv CG, Miao DL, Zhu ZG, Wu Q, Wang YG, Chen L: Development and validation of a nomogram for predicting survival in patients with gastrointestinal stromal tumours. Eur J Surg Oncol 2018, 44(10):1657-1665.

30. Shen W, Sakamoto N, Yang L: Melanoma-specific mortality and competing mortality in patients with nonmetastatic malignant melanoma: a population-based analysis. BMC Cancer 2016, 16:413.

31. Elwood M. Critical appraisal of epidemiological studies and clinical trials: Oxford University Press 2017.

\section{Figures}


Patients diagnosed with first primary lung ASC (site ICD-O-3: Lung and Bronchus; histology code: $8560 / 3$ ) during 2004 and 2015 from 18 SEER registries (1975-2016 dataset) $(\mathrm{N}=4,856)$

Exclude patients with unknown race, marriage, tumor location, and tumor laterality $(\mathrm{N}=4,280)$

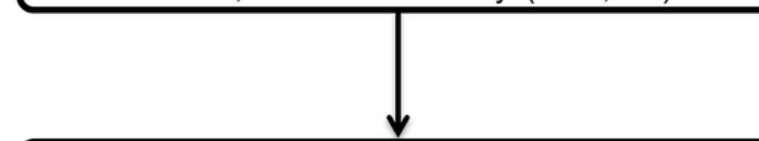

Exclude patients without active follow-up, and

without microscopical confirmation $(\mathrm{N}=4,263)$

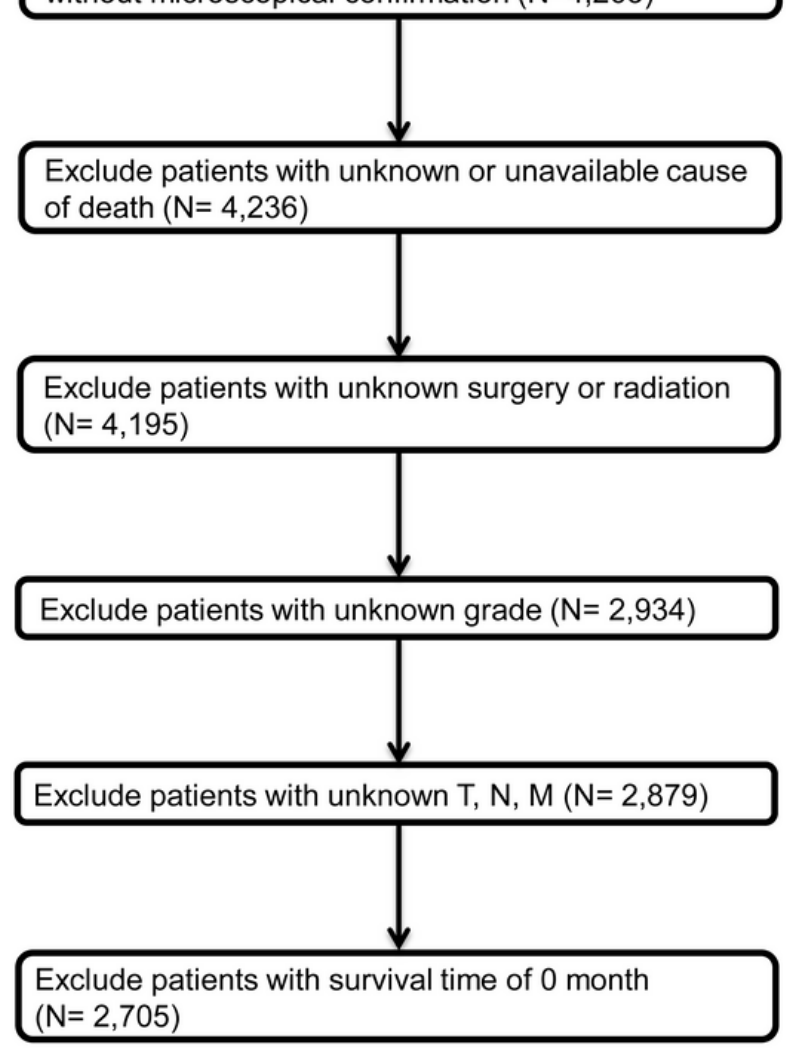

\section{Figure 1}

Flow diagram presenting the screening process in the SEER database. 

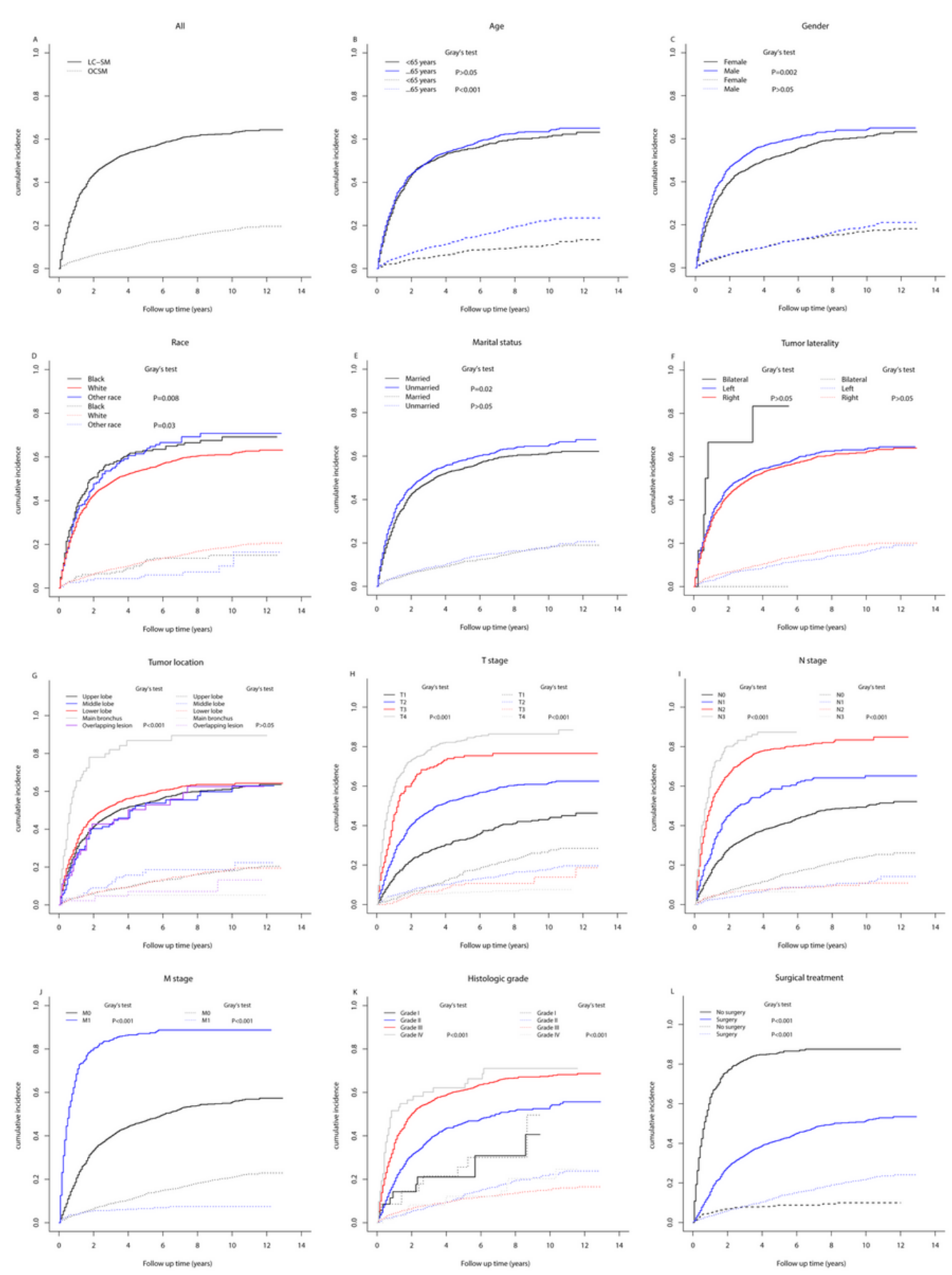

Figure 2

Cumulative incidence function curves of death for patients with lung ASC by different characteristics (solid line: LC-SM; dotted line: OCSM). Abbreviations: ASC: adenosquamous carcinoma; LC-SM: lung cancer-specific mortality; OCSM: other cause-specific morality. 
A

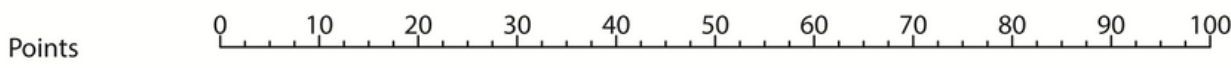

Age(years)

Sex

Surgery

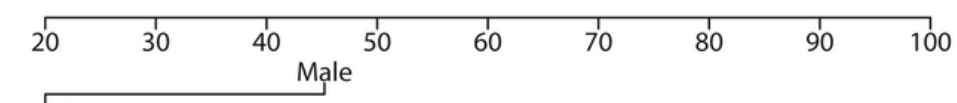

Tstage

Female

T stage

$\mathrm{N}$ stage

M stage

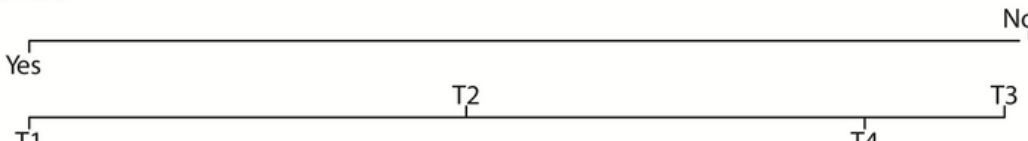

Total Points

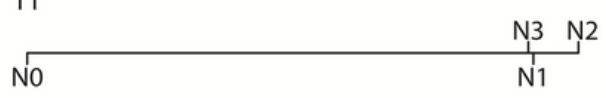

M1

3-year LC-SM

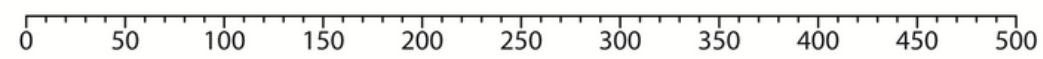

$\begin{array}{lllllllllll}0.2 & 0.3 & 0.4 & 0.5 & 0.6 & 0.7 & 0.8 & 0.9 & 0.95 & 0.99 & 0.999\end{array}$

5-year LC-SM

$\begin{array}{lllllllllll}0.2 & 0.3 & 0.4 & 0.5 & 0.6 & 0.7 & 0.8 & 0.9 & 0.95 & 0.99 & 0.999\end{array}$

B

Points

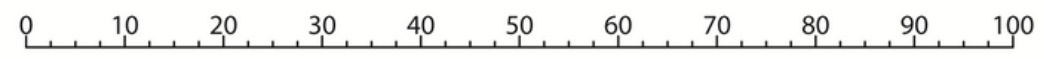

Age(years)

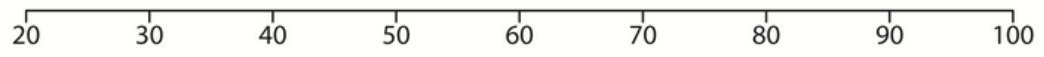

Surgery

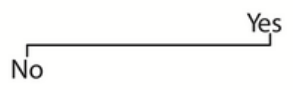

Total Points

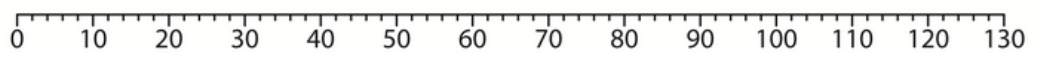

3-year OCSM

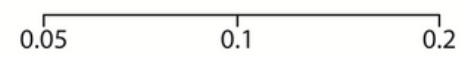

5-year OCSM

\begin{tabular}{llll}
\hline 0.05 & 0.1 & 0.2 & 0.3
\end{tabular}

Figure 3

Competing risk nomogram predicting 3-year and 5-year cumulative probabilities of death for LC-SM and OCSM among patients with lung ASC. Notes: (A) LC-SM; (B) OCSM. Abbreviations: ASC: adenosquamous carcinoma; LC-SM: lung cancer-specific mortality; OCSM: other cause-specific morality. 
A

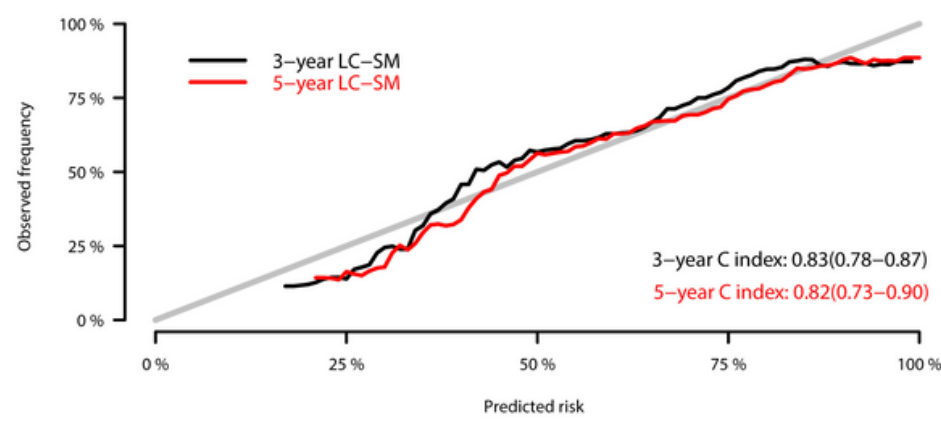

C

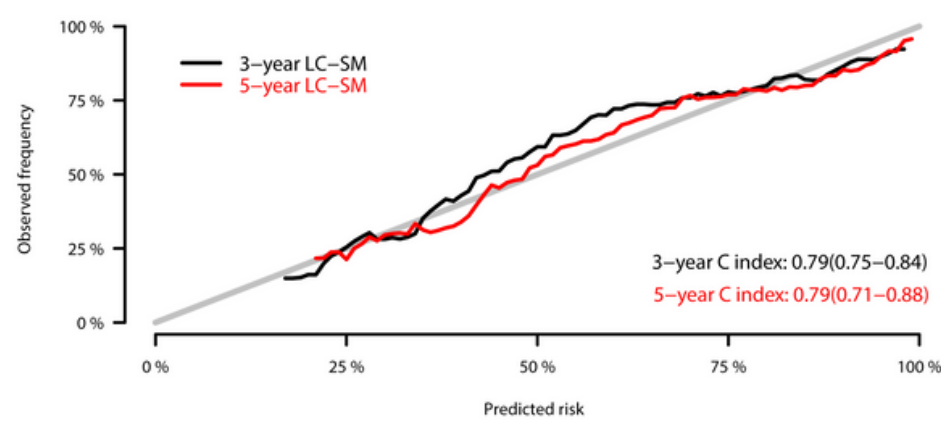

B

Calibration for training cohort

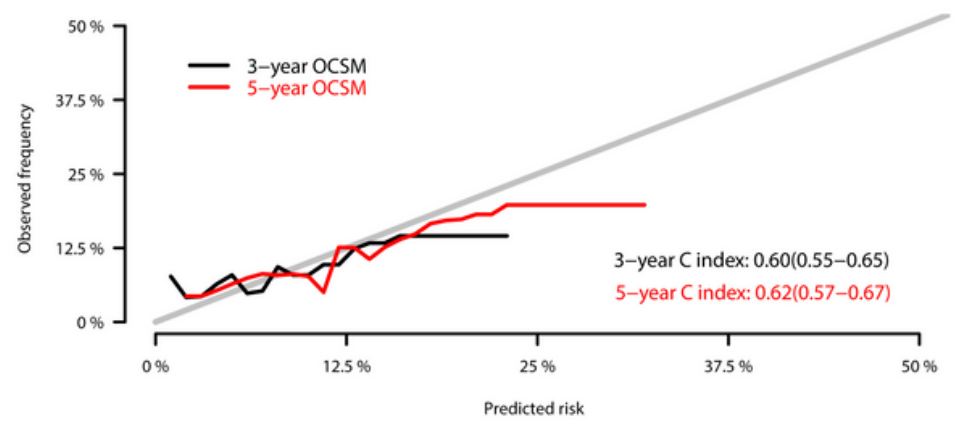

D

Calibration for validation cohort

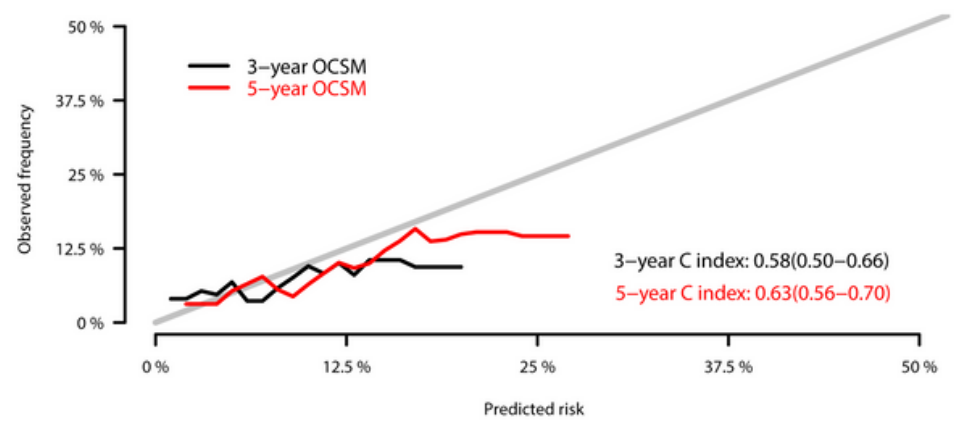

\section{Figure 4}

The 3-year and 5-year calibration curves accompanied with C-indexes for training cohort and validation cohort. The Xaxes represents the mean predicted mortality probability according to the prediction model. The Y-axes represents the observed cumulative incidence of mortality. The grey diagonal line indicates equality between the predicted and observed values. Abbreviations: LC-SM: lung cancer-specific mortality; OCSM: other cause-specific morality. 